\title{
PRÁTICAS DE MERCADO E INOVAÇÃO: DIMENSÕES ESQUECIDAS
}

\author{
Juliana Medeiros \\ Doutoranda em Administração pela Universidade Federal do Paraná - UFPR \\ Professora da Universidade Federal do Paraná - UFPR \\ julianamedeiros84@gmail.com
}

\section{Francisco Giovanni David Vieira}

Doutor em Ciências Sociais pela Pontifica Universidade Católica - PUC-SP

Professor do Departamento de Administração e do Programa de Pós-Graduação em Administração da Universidade Estadual de Maringá - UEM

fgdvieira@yahoo.com

\section{Vitor Koki da Costa Nogami}

Doutorando em Administração pela Universidade de São Paulo - FEA-USP

Pesquisador do Grupo Interdisciplinar de Pesquisas e Estudos em Marketing - GIPEM

vitornogami@gmail.com

\section{RESUMO}

Ao se fazer uma análise da literatura de marketing e inovação é possível perceber que a inovação tem como foco apenas a gestão interna da organização. Entretanto, é preciso compreender que os mercados são dinâmicos e complexos e que diversos atores que possuem crenças e valores diferentes operam em conjunto por meio de práticas que modelam tais mercados. Inovações permitem desestabilizar estruturas que estão equilibradas, gerando externalidades e fazendo com que novos modos de troca sejam definidos, bem como novas regras e regulamentações tenham que ser criadas. Essas novas definições ocorrem por meio de práticas e representações realizadas por agentes, com conhecimento especializado em marketing, que interferem nas ações de outros agentes. Nesse sentido, o presente estudo tem como objetivo, apresentar como a inovação tem sido abordada pelos pesquisadores para a construção do conhecimento da área, por meio uma análise de 1102 artigos publicados em periódicos e anais de eventos, nacionais e internacionais, demonstrando como têm sido negligenciadas dimensões presentes no processo de inovação. Para tanto, a discussão foi suportada por extensa revisão da literatura nacional e internacional sobre inovação. Como resultados o artigo apresenta proposições teóricas que podem contribuir para uma visão mais holística do fenômeno, seja do ponto de vista gerencial ou teórico.

Palavras-chave: Gestão de marketing; Práticas de mercado; Processo de inovação. 


\section{INTRODUÇÃO}

A literatura de gestão de marketing, convencionalmente, tem abordado a inovação como um processo de melhoria e desenvolvimento de novos produtos, inovação no processo de desenvolvimento do produto e na estrutura organizacional, entre outros aspectos que se relacionam às modificações internas da organização para atender o mercado e à demanda (Duin; Ortt, 2008). Nesse sentido, Araujo, Finch e Kjellberg (2010) apontam que os mercados são analisados apenas como fundos passivos e as organizações destinam-se a trabalhar seus processos inovadores agindo unicamente em melhores formas de atrair o consumidor.

A atenção que pesquisas têm dado às investigações sobre a inovação é reflexo da literatura encontrada sobre o assunto, a qual é apresentada de modo generalizado para qualquer tipo de empresa e contexto. O conhecimento disponível sobre gestão de marketing se apoia, na maioria das vezes, em pressupostos da economia, que nem sempre consideram o mercado como um campo de interações entre diversos agentes, porém, com valores específicos dentro de cada contexto. "Paradoxalmente, os mercados estão em todos os lugares, e ao mesmo tempo, em lugar nenhum" (Araujo et al., 2010). Consequentemente, as análises e compreensões da inovação como uma ferramenta de marketing para as organizações não consideram as especificidades de cada contexto nos quais as organizações estão inseridas.

De acordo com Bhalla (2011), é preciso repensar a interação existente entre marketing e inovação, uma vez que os mercados não são simples mecanismos de troca e ligação entre vendedores e compradores. Os mercados são ecossistemas onde vários agentes e empresas interagem e se conectam um ao outro para alcançar seus objetivos individuais (Bhalla, 2011). De fato, é necessário utilizar-se de técnicas de marketing para atrair consumidores, que são sugeridas pela literatura corrente. No entanto, a inovação torna-se limitada quando levado em conta apenas o conhecimento disponível na literatura atual de marketing, que não considera plenamente o processo da construção social da realidade (Smelser; Swedberg, 2005).

Nesse sentido, o objetivo da presente pesquisa consiste em investigar como a inovação tem sido abordada pelos pesquisadores para a construção do conhecimento na área voltado para acadêmicos e praticantes. O artigo demonstra que dimensões presentes no processo de inovação têm sido negligenciadas na produção sobre o tema.

Revista de Administração e Inovação, São Paulo, v. 10, n.2, p.238-261, abr./jun. 2013. 
No sentido de sua apresentação, além desta introdução contextualizando tema e objetivo, este artigo envolve quatro seções adicionais. O design do estudo revelará a seguir como os escritos foram selecionados e analisados. Logo após são elaboradas algumas críticas decorrentes desses textos selecionados, vinculadas ao método de coleta e às categorias de análise encontradas. Em seguida os resultados são apresentados em forma de propostas, oriundas da Sociologia Econômica e das dinâmicas de Práticas de Mercado. Para fortalecer o intuito do artigo, também são apresentadas algumas proposições teóricas. Por fim, as considerações finais relacionam limitações deste estudo, bem como sugestões para novas pesquisas, embasadas nos resultados encontrados e proposições realizadas.

\section{DESIGN DO ESTUDO}

A percepção sobre como a inovação tem sido estudada, derivou-se de um levantamento realizado em 14 periódicos internacionais, 8 periódicos nacionais e 5 anais de eventos nacionais, resultando em 1102 artigos. O recorte temporal selecionado foi o intervalo de cinco anos entre 2006 e 2010 por apresentarem discussões, dados e informações mais atuais e serem estimulados pelo CNPq e CAPES em termos de revisão e produção acadêmica.

Quanto aos periódicos internacionais, foram selecionados aqueles que possuem relação direta com as áreas de Inovação, Marketing e Empreendedorismo. Inovação, pois é o tema central deste levantamento. Marketing, pois a análise e os resultados serão trabalhados sob a perspectiva de Marketing e Mercado, uma vez que possui relação estreita com a área de Inovação. Por fim, Empreendedorismo, pois este campo do conhecimento também possui relação estreita com as áreas de Inovação e Marketing. Este critério também ocorreu na seleção dos anais dos eventos. Já em relação aos periódicos nacionais, como ainda não é comum encontrar periódicos com temas específicos no Brasil, foram selecionados os que apresentam maior difusão no meio acadêmico nacional. No entanto, cabe ressaltar que as três áreas do conhecimento foram selecionadas apenas como fonte de pesquisa e não como literatura base.

Depois de selecionados os periódicos e anais de eventos, os artigos foram selecionados a partir da temática de inovação. Os trabalhos que continham o termo 'inovação' no título, nas palavras chave ou no resumo, foram selecionados para análise. Deste modo, ao todo foram encontrados 1102 artigos.

Revista de Administração e Inovação, São Paulo, v. 10, n.2, p. 238-261, abr./jun. 2013. 
O Quadro 1, a seguir, aponta quais periódicos e anais de eventos que compuseram o levantamento, bem como o número de artigos analisados por publicação:

\begin{tabular}{|c|c|c|c|c|c|}
\hline $\begin{array}{l}\text { Periódicos } \\
\text { Internacionais }\end{array}$ & $\begin{array}{c}\text { Número } \\
\text { de } \\
\text { artigos }\end{array}$ & Periódicos Nacionais & $\begin{array}{c}\text { Número } \\
\text { de } \\
\text { artigos }\end{array}$ & Anais & $\begin{array}{c}\text { Número } \\
\text { de } \\
\text { artigos }\end{array}$ \\
\hline $\begin{array}{l}\text { Journal of } \\
\text { Entrepreneurship }\end{array}$ & 20 & $\begin{array}{l}\text { Revista de Administração de } \\
\text { Empresas (impressa) }\end{array}$ & 16 & EnANPAD & 89 \\
\hline $\begin{array}{l}\text { European Journal of } \\
\text { Marketing }\end{array}$ & 42 & $\begin{array}{l}\text { Revista de Administração de } \\
\text { Empresas (eletrônica) }\end{array}$ & 67 & EMA & 10 \\
\hline $\begin{array}{l}\text { European Journal of } \\
\text { Innovation } \\
\text { Management }\end{array}$ & 165 & $\begin{array}{l}\text { Revista de Administração da } \\
\text { Universidade de São Paulo }\end{array}$ & 42 & 3ES & 18 \\
\hline $\begin{array}{l}\text { Internacional Journal } \\
\text { of Innovation } \\
\text { Management }\end{array}$ & 74 & $\begin{array}{l}\text { Revista de Administração } \\
\text { Contemporânea }\end{array}$ & 20 & EnADI & 9 \\
\hline $\begin{array}{l}\text { Journal of Product } \\
\text { Innovation } \\
\text { Management }\end{array}$ & 136 & $\begin{array}{l}\text { Revista de Administração } \\
\text { Mackenzie }\end{array}$ & 20 & EGEPE & 31 \\
\hline $\begin{array}{l}\text { Journal of Business } \\
\text { Research }\end{array}$ & 31 & $\begin{array}{l}\text { Revista Eletrônica de Gestão } \\
\text { Organizacional }\end{array}$ & 15 & & \\
\hline $\begin{array}{l}\text { Journal of Marketing } \\
\text { Management }\end{array}$ & 18 & Cadernos EBAPE & 10 & & \\
\hline $\begin{array}{l}\text { Journal of Marketing } \\
\text { Theory and Practice }\end{array}$ & 9 & $\begin{array}{l}\text { Revista Eletrônica de } \\
\text { Administração }\end{array}$ & 50 & & \\
\hline $\begin{array}{l}\text { Journal of Marketing } \\
\text { Research }\end{array}$ & 12 & & & & \\
\hline $\begin{array}{l}\text { Journal of Research in } \\
\text { Marketing and } \\
\text { Entrepreneurship }\end{array}$ & 7 & & & & \\
\hline $\begin{array}{l}\text { Journal of Small } \\
\text { Business Management }\end{array}$ & 40 & & & & \\
\hline $\begin{array}{l}\text { Journal of the Academy } \\
\text { of Marketing Science }\end{array}$ & 97 & & & & \\
\hline Journal of Marketing & 33 & & & & \\
\hline $\begin{array}{l}\text { Brazilian } \\
\text { Administration Review }\end{array}$ & 12 & & & & \\
\hline
\end{tabular}

Quadro 1: Periódicos e Anais de Evento Selecionados.

Fonte: Coleta de dados, 2011.

Devido à quantidade de trabalhos encontrados, a temática da inovação foi categorizada em cinco classificações, de acordo com a análise do levantamento realizado:

C1 - Inovação em Produtos e Serviços

C2 - Atividades Organizacionais que favorecem a Inovação

C3 - Adoção e Difusão da Inovação

Revista de Administração e Inovação, São Paulo, v. 10, n.2, p.238-261, abr./jun. 2013. 
C4 - Índice de Inovação

C5 - Agentes de Mercado e Inovação

Os artigos analisados foram alocados dentro destas categorias para a realização da análise. Tal alocação resultou em uma frequência de assuntos, como pode ser observado por meio do Gráfico 1.

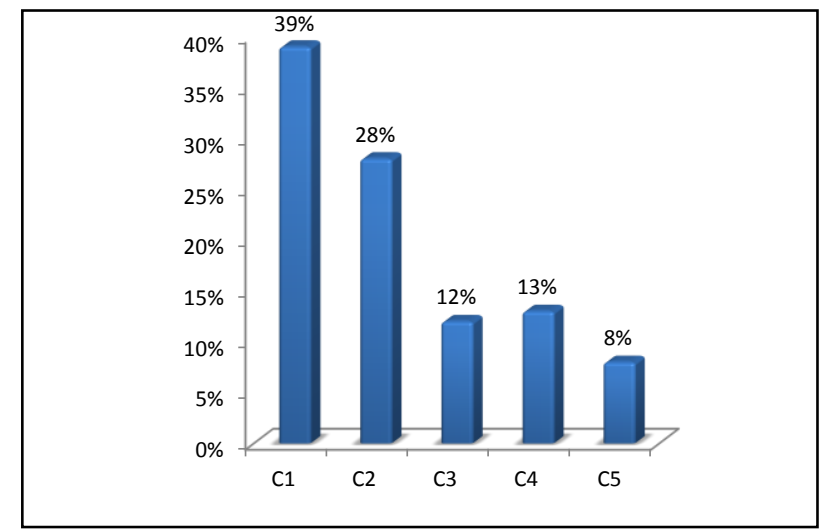

Gráfico 1: Frequência dos artigos por categoria.

Fonte: Coleta de dados, 2011.

Dentre estas categorias, não foram encontrados alguns elementos fundamentais para a inovação no mercado. Desta maneira, a análise dos artigos selecionados foi realizada a partir destas lacunas, buscando encontrar como preencher este gap teórico. Toda a seção 4 buscará apresentar os possíveis preenchimentos destes gaps. Já a seção 3 a seguir, apresenta o conteúdo encontrado no levantamento.

\section{INÍCIO DAS DISCUSSÕES SOBRE MARKETING E INOVAÇÃO}

Inovação sempre esteve presente na literatura de marketing. O processo de inovação envolve distintas definições comumente utilizadas na literatura: inovação como invenção, novas ideias - novos produtos e/ou serviços; e inovação como processo de desenvolvimento e implementação de novas ideias (Van de Ven, 1986).

Trabalhos como o de Levitt (1960) "Miopia de Marketing" e o de Drucker (1954) "The practice of management" foram fundamentais para o desenvolvimento da literatura de marketing. Com a evolução da disciplina de Marketing, a inovação sempre foi utilizada como base para a discussão das estratégias competitivas das organizações, uma vez que inovação está intimamente ligada ao desenvolvimento de produtos para atender necessidades dos consumidores (Luckas; Ferrell, 2000). No

Revista de Administração e Inovação, São Paulo, v. 10, n.2, p. 238-261, abr./jun. 2013. 
início, o foco dos pesquisadores voltava-se para a competitividade das organizações frente à concorrência apresentada no mercado, que aumentava rapidamente. As estratégias eram basicamente voltadas para as estruturas produtivas, ou seja, otimização dos processos, redução de custos e maximização dos lucros das organizações (Sassateli, 2010). As organizações buscavam inovar e desenvolver novos produtos de acordo com suas necessidades internas.

Com a publicação do artigo de Theodore Levitt em 1960, intitulado como "Marketing Myopia”, houve algumas mudanças no conhecimento do campo, pois o autor argumentava que o desempenho negativo das organizações ocorria devido à preocupação com seus produtos e suas estruturas e não com o mercado, ou seja, com o consumidor (Wright, 2002). Levitt alegava que as empresas deviam pensar em si não no sentido de produção de produtos, mas como criação de satisfação para o consumidor.

Outro autor fundamental que contribuiu para o campo de desenvolvimento da literatura de marketing e inovação foi Peter Drucker. O autor já escrevia sobre inovações que ainda nem tinham acontecido e como as organizações poderiam identificá-las. Drucker (2002) argumentava que os executivos precisavam entender a inovação como meio para explorar as mudanças. Segundo o autor, as oportunidades para a inovação podem ser identificadas pela percepção de incongruências, pela necessidade no processo, pela mudança na estrutura da indústria e do mercado, pela mudança na percepção, pela mudança demográfica e por meio da introdução de novos conhecimentos.

Na busca pela reunião das definições de marketing, Croiser (1975), compilou uma lista de 50 diferentes definições, que foram classificadas em quatro categorias: orientação para clientes; marketing como estratégia; técnicas de marketing; e inteligência de mercado (Kohli; Jaworki, 1990). Resumidamente, os livros textos da área afirmam que é necessário compreender as necessidades dos consumidores por meio de pesquisas de mercado formal, definir os segmentos que serão alvos da organização, desenvolver o produto de acordo com as necessidades identificadas, posicionando-o por meio de técnicas de preço, distribuição e atividades promocionais (Stokes, 2000).

Fundamentados por estas premissas e por métodos de pesquisas que podem limitar algumas compreensões, pesquisadores frequentemente buscam compreender como é a realidade das organizações. No entanto, as investigações e atividades desenvolvidas por praticantes de marketing focam nas inovações no sentido de melhorias e desenvolvimento de produtos; no processo interno; nas formas de comunicação; entre outras. Isso ocorre devido à literatura que orienta as pesquisas. Os conceitos basicamente apresentam formas de conectar a organização com o consumidor em uma 
dimensão bilateral, como se pudesse enquadrar em todos os tipos de negócios e contextos (Araujo et al., 2010).

\subsection{Métodos utilizados para construção da literatura em Marketing e Inovação}

Devido às origens da literatura de marketing e inovação, frequentemente as investigações ocorrem por meio de métodos que avaliam variáveis de causa e efeito, os quais não permitem observar aspectos contextuais específicos de cada organização. Como consequência, Hoholm e Araujo (2011) afirmam que não é possível a compreensão de como a inovação ocorre de fato, uma vez que são desconsideradas as relações inter e intraorganizacional que conectam todos os agentes, recursos e conhecimentos necessários para consolidar a inovação. De fato, relações de causa e efeito contribuem para o desenvolvimento do conhecimento, mas dificilmente explicam toda a complexidade da inovação no contexto social.

Ademais, o olhar foca-se apenas no momento presente, não observando como o processo de inovação pode ser construído socialmente levando em conta a conexão entre diversos agentes que contribuem para o desempenho organizacional (Hoholm; Araujo, 2011). Desse modo, as organizações acabam executando apenas práticas rotineiras seguindo modelos e desconsiderando os valores, regras, crenças e atitudes de determinada sociedade.

Dentre as publicações levantadas nos últimos cinco anos, em periódicos nacionais e internacionais, foi significativa a utilização de métodos que não permitem a visualização do processo de inovação como um todo. Muitos trabalhos publicados utilizaram métodos quantitativos e estudos de casos, e os poucos que utilizaram métodos qualitativos e trataram os dados por meio de métodos interpretativos, tinham como unidade de análise a organização e o consumidor. Em outras palavras, em praticamente nenhum destes artigos analisados, foi notada a preocupação com outros agentes, como intermediários, governos, sindicatos, imprensa e outros que também podem contribuir no processo de inovação.

De fato, métodos quantitativos apresentam vantagens no sentido de representar melhor um universo. No entanto, limita a compreensão da complexidade do processo e das relações envolvidas, desconsiderando questões contextuais como valores, crenças, regras, significados de cada agente da operação e de cada ambiente específico, que impactam suas atividades e modos de agir (Hoholm e Araujo, 2011).

Revista de Administração e Inovação, São Paulo, v. 10, n.2, p. 238-261, abr./jun. 2013. 
Por outro lado, estudo de caso é um método que permite a complementariedade de diversas formas de tratar os dados, tanto quantitativamente, quanto qualitativamente. Entretanto, muitos estudos de caso são estáticos e se concentram em representações de variáveis como "culturas internas" e “estruturas internas", não considerando outros aspectos (principalmente externos) que impactam nas ações das organizações (Hoholm e Araujo 2011).

\subsection{Categorias de pesquisa}

Segundo Cahill (1996), pesquisadores têm buscado generalizar o fenômeno da inovação. Isso ocorre devido a dois fatores: (a) a utilização de métodos de pesquisa que pouco permitem a compreensão de aspectos presentes em diferentes contextos; e (b) a grande preocupação em descobrir quais elementos impactam no processo de inovação, focando apenas internamente na organização. Ao revisar a literatura dos últimos cinco anos (nacional e internacional), nota-se que os estudos se apresentam de modo a demonstrar modelos de gerenciamento de inovações por meio de melhores técnicas de gestão (Duin; Ortt, 2008).

Deste modo, segue a categorização realizada a partir dos assuntos encontrados no levantamento da pesquisa:

\section{C1 - Inovação em Produtos e Serviços}

Na categoria de inovação em produtos e serviços, foram encontradas pesquisas relacionadas a: (a) fatores que levam ao sucesso dos novos produtos (Bailetti; Taney, 2008; Calantoni, 2010; Klink; Athaide, 2010); (b) imitação e inovação (Kalwani et. al., 2006; David et. al., 2008); (c) valor da marca (Sorescu; Spanjol, 2008); (d) desenvolvimento de produtos (Joshi, 2009; Silva et. al., 2008; Cruz; Delgado, 2009; Garcez; Wright, 2010); (e) participação dos consumidores na inovação de produtos (Dankbaar; Janssen, 2008; Evans, 2008); e (f) comunicação, embalagens e design (Lutz et. al., 2009; Beverland et. al., 2010).

No entanto, inovar em produtos e serviços implica uma ampla gama de recursos (Gonçalves Filho; Gonçalve; Pardini, 2008; Moretti; Moysés Filho; Pereira, 2010; Caetano; Kuromoto; Amaral, 2012) que muitas vezes não é de posse apenas de uma única organização e/ou individuo (Hoholm; Araujo, 2011). Inovar pode envolver diversos agentes que com suas ações impactam no processo podendo direcionar o mesmo para lados distintos (Neyland; Simakova, 2010). Isso dependerá do contexto e cultura que permeia o mercado onde a inovação será desenvolvida e difundida (Araujo et

Revista de Administração e Inovação, São Paulo, v. 10, n.2, p.238-261, abr./jun. 2013. 
al., 2010). Ao tratar de atributos intangíveis como marca do produto, o contexto e a cultura apresentam-se, ainda, como fatores mais pontuais, uma vez que o significado de uma marca para os agentes de uma região pode mudar quando esta marca é comunicada em outra região. Deste modo, a participação dos consumidores e de outros agentes no processo de inovação, dependerá da realidade construída socialmente dentro de determinada sociedade.

\section{C2 - Atividades Organizacionais que Favorecem a Inovação}

Nesta categoria, os fatores que influenciam a inovação de acordo com o levantamento realizado, são: (a) estrutura organizacional (Guimaraes; Monaco, 2007; Smith et. al., 2008; Miguel; Teixeira, 2009; Porto et. al., 2010; Cakar; Erturk, 2010); (b) gestão da inovação (Berglund, 2007; Ferro et. al., 2007); (c) incentivo à criatividade (Assink, 2006; Kalling, 2007; Dobini, 2008; Baier et. al., 2008); (d) influência da liderança para inovação (Jong; Hartog, 2007; Beneditte; Torkomian, 2008); e (e) modelos de gestão que buscam identificar oportunidades inovadoras (Drew, 2006; Macgregor, 2006; Blenkinossop; Zidunczyk, 2007).

De fato, algumas atividades que são realizadas pela organização proporcionam um ambiente mais favorável à inovação, estimulando a capacidade de inovar e dar ideias. Entretanto, o olhar voltase apenas para a organização (Araujo et al., 2010). Em outras palavras a preocupação é com a cultura interna, os recursos que a organização tem disponível, a estrutura física. Valores e significados construídos por uma sociedade onde o produto circula também podem impactar no processo de inovação. A ressignificação de produtos por parte de consumidores e agentes do mercado pode proporcionar novas ideias. A estrutura do mercado como um todo (leis, regulamentações, distribuição de distribuição, governo, mídia) pode influenciar no desempenho da organização, como afirmado por Kjellberg e Helgesson (2007), frente à inovação. Produtos desenvolvidos por outras organizações podem favorecer a inovação. Deste modo, os agentes organizacionais que estejam atentos a estes sinais têm a possibilidade de melhor desempenho ao desenvolver e difundir uma inovação.

\section{C3 - Adoção e Difusão da Inovação}

No que se refere à adoção da inovação, foram encontradas pesquisas relacionadas tanto à adoção da inovação por parte dos consumidores, quanto à adoção da inovação e novas tecnologias por parte das organizações (Pereira et. al., 2007; Aguiar et. al., 2009; Antioco; Kleijnen, 2010; Perez; Zwickler, 2010).

Revista de Administração e Inovação, São Paulo, v. 10, n.2, p. 238-261, abr./jun. 2013. 
No entanto, as pesquisas trabalham em uma perspectiva unilateral quando discutem a adoção e difusão da inovação, ou seja, identificam apenas a conexão entre a organização e o consumidor (Araujo et al., 2010). No mercado, outros agentes podem interferir na adoção e difusão de uma inovação. Suas ações podem mobilizar esforços para formatar novos modelos de negócios que facilitam a entrada de novos produtos, bem como a percepção e difusão da inovação por parte de agentes mais resistentes às mudanças. Além dos esforços dos agentes, dispositivos materiais (como outros produtos) podem complementar e auxiliar propagação desta inovação (Neyland; Simakova, 2010).

\section{C4 - Índice de Inovação}

Já em relação à categoria de índice de inovação, os componentes encontrados nos artigos pesquisados são: (a) relação de dependência entre o desempenho organizacional e o índice de inovação das empresas (Meirelles et. al., 2008; Boehe et. al., 2008; Auhb et. al., 2009; Bowena, 2010); (b) relação de dependência entre a orientação da organização (para o mercado, para os concorrentes ou para os processos internos) e o índice de inovação (Paladino, 2007; Carmen; José, 2008; Laforet, 2008; Gotze et. al., 2009).

O desempenho de uma organização, entretanto, pode não depender apenas do índice de introdução de inovação. Outras variáveis podem interferir neste desempenho, como: a regulamentação existente na região onde a inovação é introduzida; a logística e estrutura física deste mercado; o significado dessa inovação para os diversos agentes do mercado, entre outras. Isso implica que, caso uma organização tenha uma orientação que proporciona alto índice de inovação, há maior probabilidade de melhor desempenho. Porém, ser orientado por uma ou outra perspectiva não garante maior índice de inovação, uma vez que, segundo Hoholm e Araujo (2011), inovar envolve recursos que podem não estar disponíveis para a organização.

\section{C5 - Agentes de Mercado e Inovação}

Por fim, na categoria de agentes de mercado e inovação os assuntos encontrados foram: (a) identificação de atores que podem contribuir para a inovação (Achterkamp; Marjolein, 2006; Doolin; Troshani, 2007; Hyysalo; Stewart, 2008; Basso et. al., 2008; Borg, 2009); e (b) adequação das inovações às regras vigentes (Dyerson; Pilkingto, 2006).

De fato, a última categoria aponta indícios da mudança de foco nas pesquisas permeando assuntos sobre redes e regulamentações que podem influenciar as inovações das organizações. No

Revista de Administração e Inovação, São Paulo, v. 10, n.2, p.238-261, abr./jun. 2013. 
entanto, quando discutem redes, abordam a questão da identificação de atores que podem contribuir para a inovação nas organizações, sem discutir aspectos como o significado das ações dos agentes das redes, valores e crenças que influenciam estas ações e contexto em que as redes estão inseridas, entre outros aspectos (Araujo et al., 2010; Hagberg; Kjellberg, 2010). Quando as pesquisas trazem à discussão regras e regulamentações do mercado, discutem como e não chama a atenção dos agentes do mercado para a formatação das regras por meio de suas forças representativas (Kjellberg; Helgesson, 2007).

Neste sentido, ao analisar essas categorias verifica-se que é negligenciada a construção social que ocorre na prática e a complexa dinâmica de mercado. São esquecidas algumas dimensões, que podem auxiliar na construção de redes de relacionamento entre os diversos atores de mercado que poderão influenciar, direta ou indiretamente, o processo de inovação. Ficam esquecidas, também, as especificidades de cada contexto em que a organização está inserida. Isso possivelmente ocorre devido ao fato de que é utilizada uma literatura que permite enquadrar as atividades realizadas pela organização em qualquer ambiente.

\section{REORIENTANDO O OLHAR PARA O PROCESSO DE INOVAÇÃO}

Para que seja possível compreender o processo de inovação, é preciso se afastar da literatura convencional e observar a inovação como um processo construído socialmente. No entanto, pressupostos da economia dificilmente explicam esse processo de construção social, uma vez que, de forma determinista, consideram os mercados estáveis e como se fossem "dados" não passíveis de modificações por meio de esforços conjuntos (Araujo et al., 2010).

Para os pesquisadores e praticantes da visão econômica, que deram origem à literatura de marketing e inovação, os agentes trabalham única e exclusivamente nas relações de compra e venda (Araujo et al., 2010), maximizando atividades e comportamentos que atinjam os objetivos das organizações: gerar lucro. Este tipo de atividade envolve apenas trabalhar com desenvolvimento de novos produtos por meio da identificação de oportunidade para inovação, posicionamento e técnicas de marketing para atrair o consumidor e buscando ser competitivo por meio da redução de custos e consequentemente menor preço (Swedberg, 2003).

Essa visão direciona o pensamento para a realização de atividades baseadas em cálculos racionais, desconsiderando a cultura, os valores e as crenças dos agentes da operação. As trocas e os mercados são considerados estáveis e, portanto, modelos e teorias podem ser generalizadas para

Revista de Administração e Inovação, São Paulo, v. 10, n.2, p. 238-261, abr./jun. 2013. 
qualquer tipo de contexto e organização (Araujo, et. al. 2010). Neste sentido, esta seção apresenta o olhar que pode ser dado ao processo de inovação, que permite analisar e compreender dimensões que nem sempre são avaliadas e que podem interferir em tal processo.

\subsection{Contribuições da sociologia econômica}

A perspectiva da Sociologia Econômica considera o mercado como uma instituição, um campo composto por agentes que se relacionam e interferem ativamente no processo de inovação. Estes agentes se relacionam dentro do mercado na finalidade de efetuar trocas econômicas (Hagberg; Kjellberg, 2010; Swedberg, 2003). O contexto influencia as relações e ações (Araujo et al., 2010). Em outras palavras, as interações serão influenciadas pela estrutura do mercado, valores, crenças e significados que direcionam as atividades e ações de cada indivíduo que está inserido no mercado. Sendo assim, o mercado se torna um resultado não intencional de ações intencionais.

De acordo com Swedberg (2003) e Granovetter (1985), os mercados estão incluídos em redes sociais que afetam os resultados econômicos. Essas redes precisam ser identificadas e compreendidas não apenas pela quantidade de agentes, nem pela disposição e alcance de cada um dentro da rede, mas também os significados que são atribuídos em cada uma das ações dos agentes devem ser analisados. Isso ocorre, devido ao fato de que a ação de cada agente pode interferir na ação de outros e resultar na inovação bem sucedida (Burt, 2004). É preciso compreender que inovações podem ter melhor desempenho dentro de um mercado, caso haja convergência de esforços dos diversos agentes para que seja estabilizada a nova forma de troca introduzida pela inovação.

Nesse sentido, é preciso analisar os mercados de forma diferente da literatura convencional de marketing e inovação. Tal literatura representa os mercados como fundos passivos e considerados como praticamente inexistentes. O foco é apenas na interação entre a organização e os consumidores, bem como nas formas de organizar as trocas econômicas entre os mesmos (técnicas de gestão e composto de marketing), negligenciando a existência de qualquer outro tipo de agente que possa influenciar tal troca (Araujo et al., 2010). Com isso, o mercado e as necessidades de recursos e conhecimentos que as inovações precisam nem sempre são levados em consideração. Organizações inovadoras dificilmente possuem todos os recursos, tecnologia e conhecimento suficiente para a consolidação da inovação ou da ideia. Isso significa que a rede de agentes é crucial para o novo processo operacional, bem como para introduzir o novo produto (Hoholm; Araujo, 2011). Assim, esta

Revista de Administração e Inovação, São Paulo, v. 10, n.2, p.238-261, abr./jun. 2013. 
literatura torna-se limitada para a análise e compreensão de como de fato ocorre o processo de inovação.

Tendo em vista a discussão até aqui realizada, e com base no que apontam Campanario et. al., (2012) e Campanario e Chagas Jr. (2012), seguem as proposições teóricas PT 1, PT 2, PT 3 e PT 4:

PT 1: A concepção da ideia inovadora é condição necessária, mas não suficiente para que seja consolidada a inovação.

PT 2: O processo de inovação, desde a concepção inicial até a sua adoção, tem a possibilidade de ser mais bem sucedido quando mais de um agente dispende esforços para consolidá-la.

PT 3: Compreender o processo de inovação implica entender o significado das ações de cada agente dentro do mercado.

PT 4: Inovações bem sucedidas são resultado da compreensão da construção social dos significados, valores e cultura de uma sociedade.

\subsection{Dimensões esquecidas: dinâmica e práticas de mercado}

Para que se possa compreender o processo de inovação é preciso enfrentar os desafios que podem ser encontrados na área de Marketing e Inovação, os quais foram descritos por Hoholm e Araujo (2011): a) utilizar métodos de estudos que permitam investigar os agentes, suas inter-relações e os significados de suas ações; b) compreender a dinâmica que ocorre no processo de inovação, a qual transcende o simples mapeamento da estrutura ou descobertas da forma de produzir a inovação e suas relações de causa e efeito; e c) descrever o processo e sua ordenação, indo além de apenas contabilizar os fatos.

Inúmeras características generalizadas têm sido identificadas no processo de inovação (Hoholm; Araujo, 2011). Contudo, sabe-se que a inovação opera em ambientes contingentes, alterando as propriedades dos agentes e dos modos de troca (Araujo, 2007; Callon, 2010; Kjellberg; Helgesson, 2007). No entanto, frequentemente tem sido esquecida a complexidade da dinâmica do mercado e as práticas que podem ser executadas, as quais modelam mercados. Essa dinâmica envolve quatro premissas: 1) mercados são híbridos e coletivos (Neyland; Simakova, 2010); 2) conhecimento especializado em marketing é performativo e formata mercados (Hagberg; Kjellberg, 2010); 3) a construção dos mercados envolve framing (enquadramento de regras e regulamentações) e overflowing (externalidades) (Callon, 1998); e 4) mercados são resultados de práticas (Kjellberg; Helgesson, 2007).

Revista de Administração e Inovação, São Paulo, v. 10, n.2, p. 238-261, abr./jun. 2013. 
A primeira premissa aborda o mercado como híbrido e coletivo, pois não são compostos apenas por organização e consumidores (Neyland; Simakova, 2010). Híbridos, pois agentes humanos e dispositivos materiais em conjunto podem contribuir na formatação do mercado quando uma inovação é introduzida (Neyland; Simakova, 2010). Em outras palavras, o processo de inovação não depende apenas de uma grande ideia e conhecimento, mas também de tecnologias, recursos, entre outros atores e dispositivos. Coletivos, pois uma estrutura de mercado é composta por inúmeros agentes que estão conectados. Estes agentes precisam trabalhar em conformidade nas suas ações para que haja estabilização da estrutura, posterior ao desequilíbrio gerado pela inovação (Hagberg; Kjellberg, 2010).

A segunda premissa aponta que é necessário que os agentes possuam conhecimento especializado em marketing o que os leva a identificar atores dentro das redes, bem como sua estrutura e suas inter-relações (Hagberg; Kjellberg, 2010). Esta capacidade permite que o agente ou a organização identifique o papel que deve ser desempenhado por cada um dentro da rede para que a inovação seja consolidada. Deste modo, é possível que haja a interferência dos agentes com conhecimento especializado que direcionam as ações daqueles que não estão em conformidade para que haja novamente o equilíbrio dentro do mercado e desempenho coletivo (Kjellberg; Helgesson, 2007).

A terceira premissa considera que modificações no mercado não ocorrem apenas dentro de um framing, ou seja, não altera apenas as formas de trocas entre a organização e o consumidor (Callon, 1998). Toda a estrutura é desequilibrada gerando externalidades (Callon, 1998). Isso significa que a introdução de uma inovação ultrapassa a "moldura" que abrange apenas as relações entre a organização e o consumidor, a qual envolve apenas regras, normas e contratos (formais e informais) e atinge o overflowing (Araujo et al., 2010; Callon, 1998). Ultrapassar a "moldura" representa gerar externalidades em todo o ambiente que estava estabilizado. Em outras palavras, são alteradas as formas de distribuição, são formatadas novas regras e regulamentos, são definidas novas formas de realizar trocas e de se relacionar com os diversos agentes, entre outros aspectos.

Sendo assim, a quarta premissa aponta que os mercados podem ser remodelados, ou construídos e reconstruídos, continuamente, com a introdução de inovação seguida de esforços de agentes que possuem interesses comuns (Kjellberg; Helgesson, 2006). Estes esforços podem aqui ser compreendidos como práticas de mercado. O mercado é instável e mudanças acontecem frequentemente. Enquanto há ações dentro da estrutura do mercado, a estrutura em si está sendo processada e essas mudanças impactam as ações futuras. Desse modo, é preciso considerar que há uma

Revista de Administração e Inovação, São Paulo, v. 10, n.2, p.238-261, abr./jun. 2013. 
interação entre a estrutura do mercado e o processo de ações dos agentes para alcançar seus objetivos próprios (Kjellberg; Helgesson, 2007b).

Tais práticas formam um tríplice, como sugeridas por Kjellberg e Helgesson (2007a): práticas de troca; práticas representacionais e práticas normativas, conforme se observa na Figura 1.

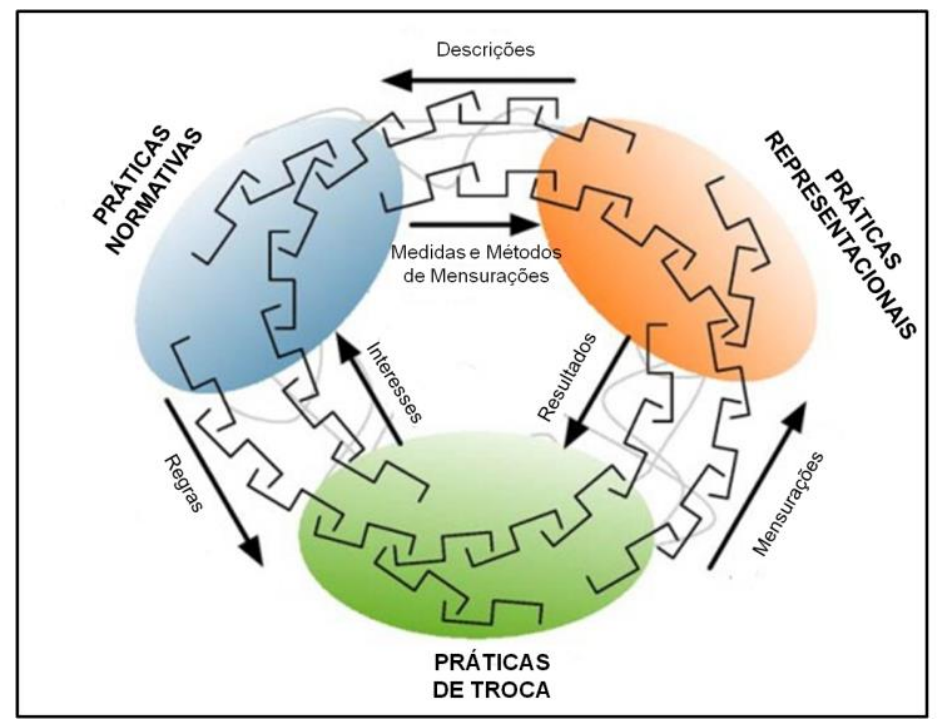

Figura 1: Transações e Intermediários na Prática de Mercado. Fonte: Kjellberg e Helgesson, 2007a, p. 151.

Práticas de troca: são práticas relacionadas ao consumo concreto da troca econômica. Isto inclui específica apresentação do produto; negociação de preço e forma de pagamento; promoção; distribuição (Kjellberg; Helgesson; 2007a). Ademais, contribui para a estabilização do novo formato de troca (Cochoy, 2010) que foi remodelado pela introdução da inovação. No entanto, não devem ser executadas de modo generalizado, como é apresentado pela literatura e defendida pela visão econômica (Araujo, 2010). Essas técnicas devem levar em consideração os significados construídos ao longo do tempo dentro de uma sociedade, os valores e crenças de um determinado ambiente, bem como a estrutura do mercado em que os agentes operam. Isso sugere que os diferentes agentes envolvidos na troca precisam ser identificados e compreendidos, pois suas atitudes levam em conta estes aspectos (Hagberg; Kjellberg, 2010).

Práticas representacionais: são práticas que contribuem para descrever mercados, bem como descrever como eles funcionam. Mercados são entidades abstratas e é necessário fazer a ligação das distâncias de espaço e tempo entre as trocas e as imagens produzidas deste mercado (Kjellberg; Helgesson; 2007a). Essas práticas podem ser usadas para influenciar os outros agentes na tentativa de 
somar esforços em determinada direção (Fries, 2010). Sendo assim, o agente com conhecimento especializado em marketing (Hagberg; Kjellberg, 2010) pode representar como são os mercados por meio de estatísticas, interferindo nas práticas que serão executadas por um agente ou um grupo de agentes, bem como suas estratégias de marketing (Golfetto; Rinallo, 2006).

Práticas normativas: são práticas que permitem o estabelecimento de diretrizes para estabilizar regras e normas do mercado e como os agentes devem operar nele (Kjellberg; Helgesson; 2007). São esforços que permitem as especificações de regras e regulamentos gerais de competição e de mercado (Kjellberg, 2010). Agentes com conhecimento especializado em marketing podem agir também na esfera pública para direcionar as regras que devem limitar os modos de troca e, ainda, capacitar outros agentes para fazer o mesmo (Fries, 2010). Esses limites são fundamentais quando uma inovação é introduzida, uma vez que o ambiente está em desequilíbrio e as regras anteriores podem não se enquadrar nas novas formas de troca, facilitando atitudes oportunistas e que dificultam a estabilização e consolidação da inovação.

Deste modo, a troca é analisada não como um evento único, mas como instituição: o mercado é um cenário e a troca acontece dentro dele. Agentes com diferentes interesses se relacionam permitindo que a demanda e oferta se remodelem. Como consequência, a estrutura existente pode ser redefinida (Araujo, 2007; Callon, 2010). Isso ocorre a partir de ações de agências capacitadas, que por meio de práticas de mercado desequilibram estruturas estáveis e alteram as propriedades dos agentes e dos modos de troca quando introduzem uma inovação (Araujo, 2007; Kjellberg; Helgesson, 2007). Essas desestabilizações geram externalidades no mercado e na estrutura (overflowing), as quais extrapolam o enquadramento (framing) da organização e do consumidor (Callon, 1998).

Seguem, então, as proposições teóricas relacionadas às dimensões esquecidas:

PT 5: Para compreender como ocorre o processo de inovação, é preciso reorientar o olhar da organização para o mercado.

PT 6: O processo de inovação depende da hibridização e da coletividade dos agentes dentro do mercado.

PT 7: O agente com conhecimento especializado em marketing é capaz de compreender cada um dos agentes do mercado e interferir em suas ações.

PT 8: Inovações modificam estruturas de mercados que extrapolam o framing (enquadramento) atingindo o overflowing (externalidades), remodelando o mercado.

PT 9: Mercados são resultados de práticas de troca, representacionais e normativas.

Revista de Administração e Inovação, São Paulo, v. 10, n.2, p.238-261, abr./jun. 2013. 


\section{CONSIDERAÇÕES FINAIS}

O objetivo deste artigo foi discorrer sobre como a literatura de inovação tem sido discutida nacional e internacionalmente por meio de um levantamento que abrangeu 1102 artigos. Tal assunto tem sido abordado, na maioria das vezes, focado internamente nas organizações e por meio de relação de causa e efeito. A construção do conhecimento existente teve sua importância no desenvolvimento da área, porém apresenta dificuldades de caráter holístico para a compreensão da complexidade de uma inovação como um fenômeno social.

Sendo assim, foram apontadas algumas críticas de como o processo de inovação tem sido abordado na literatura de Inovação, Marketing e Empreendedorismo. Em seguida foram propostas abordagens baseadas na Sociologia Econômica (Granovetter, 1985; Swedberg, 2003; Hagberg; Kjellberg, 2010) e nas dinâmicas e práticas de mercado (Kjellberg; Helgesson, 2007; Hoholm; Araujo, 2011; Araujo et al., 2010) para preencher esta lacuna. Para tanto, a partir do resultado da análise do levantamento, foram apresentadas proposições teóricas a fim de instigar novas abordagens para o processo de inovação para as organizações no mercado.

Como contribuição, o artigo visou proporcionar uma visão ampla da complexidade do processo de inovação no mercado, abrindo perspectivas de análises para melhor compreensão do fenômeno. Isso poderá auxiliar em uma visão holística de como ocorre o processo de inovação, a partir da consideração de que mercados são coletivos e híbridos, possuindo diferentes verdades morais e diferentes culturas. De caráter teórico, juntamente com o conhecimento já existente, pesquisas, a partir dos pressupostos apresentados, poderão compreender e explicar de forma mais abrangente como inovações se tornam bem sucedidas. De caráter gerencial, agentes do mercado poderão visualizar como ações coletivas e análises de contextos podem levar ao desempenho positivo.

Uma limitação do trabalho está relacionada com a forma de análise deste estudo. Uma vez que foram analisados 1102 artigos foi necessário criar categorias que permitissem uma análise mais concisa. Para tanto, os artigos analisado não necessariamente discorriam sobre as categorias criadas e, assim, foi preciso definir critério para alocar cada assunto nas cinco categorias criadas, conforme foi explicado no decorrer do trabalho. Outra limitação se refere aos periódicos e anais de evento selecionados, posto que uma grande quantidade de artigos ficou fora da análise deste levantamento.

Como proposta para futuras pesquisas, este trabalho sugere a utilização das abordagens da Sociologia Econômica e das dinâmicas e práticas de mercado como base de fundamentação teórica 
para pesquisa sobre inovação. Investigar empiricamente e aprofundar teoricamente as proposições levantadas neste artigo, também é algo que pode ser realizado posteriormente.

\section{REFERÊNCIAS}

Achterkamp, M., \& Marjolein, J. (2006). Stakeholder identification in innovation projects. European Journal of Innovation Management, 9(2), 161-178.

Aguiar, L., Barcellos, M., Ferreira, G., \& Vieira, L. (2009). Willingness to try innovative food products: a comparison between British and Brazilian consumers. Brazilian Administration Review, 6(1), 50-61.

Antioco, M., \& Kleijnen, E. (2010). Consumer adoption of technological innovations. European Journal of Marketing, 44(11/12), 1700-1724.

Araujo, L. (2007). Markets, market-making and marketing. Marketing Theory, 7 (2), 211-226.

Araujo, L., Finch, J., \& Kjellberg, H. (Eds.) (2010). Reconnecting marketing to markets. Oxford: Oxford University Press.

Assink, M. (2006). Inhibitors of disruptive innovation capabitity: a conceptual model. European Journal of Innovation Management, 9(2), 215-233.

Auhb, S., Clercqa, D., \& Menguca, B. (2009). Unpacking the relationship between an innovation strategy and firm performance: the role of task conflict and political activity. Journal of Business Research, 62(11), 1046-1053.

Baier, M., Graefe, G., \& Roemer, E. (2008). Selecting promising business ideas for innovate IT services. European Journal of Innovation Management, 11(4), 560-576.

Bailetti, T., \& Taney, S. (2008). Competitive intelligence information and innovation in small Canadian firms. European Journal of Marketing, 42(7/8), 786-803.

Basso, L., Kimura, H., \& Martin, D. (2008). Redes sociais e o marketing de inovações. Revista de Administração Mackenzie, 9(1), 157-181.

Beneditte, M., \& Torkomian, A. (2008). A inovação e o papel do empreendedor na obtenção de vantagem competitiva em manufatura. In ANEGEPE (orgs). Anais do EGEPE - Encontro de Gestão de Pequenas Empresas e Empreendedorismo.

Berglund, H. (2007). Risk conception and risk management in corporate innovation: lessons from two Swedish cases. International Journal of Innovation Management, 11(4), 497-513.

Revista de Administração e Inovação, São Paulo, v. 10, n.2, p.238-261, abr./jun. 2013. 
Beverland,B., Napoli, J., \& Farrelly, F. (2010). Can all brands innovate in the same way? a typology of brand position and innovation effort. Journal of Product Innovation Management, 27.

Bhalla, G. (2011). Rethinking marketing and innovation. Em: Bhalla, G. Collaboration and cocreation: new plataforms for marketing and innovation. New York: Springer, 123-143.

Blenkinosopp, J., \& Zdunczyk, K. (2007). Do organisational factors support creativity and innovation in Polish firms? European Journal of Innovation Management, 10 (1), 25-40.

Boehe, D. (2007). Desenvolvimento de produtos em subsidiárias de empresas multinacionais no Brasil. Revista de Administração de Empresas, 47(1), 575-593.

Borg, E. A. (2009). The marketing of innovations in hight-technology companies: a network approach. European Journal of Marketing, 43(3/4), 364-370.

Bowena, F. (2010). Timing is everything: a meta-analysis of the relationships between organizational performance and innovation. Journal of Business Research, 63(12), 1179-1185.

Burt, R. (2004). Structural Holes and good Ideas. American Journal of Sociology, 110 (2).

Cahill, D. J. (1996). Pioneer advantage: is it real? Does it matter? Marketing Intelligence \& Planning, 14(4), 5-8.

Caetano, M., Kurumotto, J. S. \& Amaral, D. C. (2012). Estratégia de integração entre tecnologia e produto: identificação de atividades críticas no processo de inovação. Revista de Administração $e$ Inovação, 9(2), 124-148.

Cakar, N., \& Ertuk, A. (2010). Comparing innovation capability of small and medium-sized enterprises: examining the effects of organizational culture and empowerment. Journal of Small Business Management, 48(3), 325-359.

Callon, M. (1998). 'An essay on framing and overflowing: economic externalities revisited by sociology'. In: Callon, Michel (ed.). The laws of the market. Oxford: Basil Blackwell.

Callon, M. (1998). Introduction: the embeddedness of economic markets. In: Callon, Michel (ed.). The laws of the market. Oxford: Basil Blackwell, 1998.

Callon, M. (2010). Marketing as an art and science of market framing: commentary. In: Araujo, L., Finch, J., \& Kjellberg, H. (ed.). Reconnecting marketing to markets. Oxford: Oxford University Press. 224-233.

Calantone, R. J., Harmancioglu, N., \& Droge, C. (2010). Inconclusive innovation "returns": a metaanalysis of research on innovation in new product development. Journal of Product Innovation Management, 27(7), 1065-1081.

Campanario, M. A., \& Chagas Junior, M. F. (2012). Projetos de pesquisas puras (básicas) em marketing. In N. K. Pizzinatto, \& O. E. Farah (Orgs.), Pesquisa pura e aplicada para marketing: processos e aplicações (pp. 21-38). São Paulo: Atlas.

Revista de Administração e Inovação, São Paulo, v. 10, n.2, p. 238-261, abr./jun. 2013. 
Campanario, M. A., Chagas Junior, M. F., \& Ruiz, M. S. (2012). O modelo de Karl Popper sob a ótica das ciências sociais aplicadas. Revista de Ciências da Administração, 14(32), 124-140.

Carmen, C., \& José, G. M. (2008). The role of technological and organizational innovation in the relation between market orientation and performance in cultural organizations. European Journal of Innovation Management, 11(3), 413-434.

Cochoy, F. (2010). Reconnecting marketing to "market things": how grocery equipament drove modern consumption (progressive gorcer, 1929-1959). In: Araujo, L.; Finch, J.; Kjellberg, H. (ed.). Reconnecting marketing to markets. Oxford: Oxford University Press. 29-49.

Crosier, K. (1975). What exactly marketing? Quarterly Review of Marketing, 2(1), 173-192.

Dankbaar, B., \& Janssen, K. (2008). Proactive involvement of consumers in innovation: selecting appropriate techniques. International Journal of Innovation Management, 12(3) 511-541.

David, A., John, G., \& Oing, W. (2008). As time goes by: do cold feet follow warm intentions for really new versus incrementally new products? Journal of Marketing Research, 45(3), 307-319.

Delgado, N., \& Cruz, L. (2009). As inovações no setor de laticínios: o caso francês do grupo cooperativo $3^{\text {a }}$. Revista Eletrônica de Administração, 15(3).

Drucker, P. F. (2002). Innovation and entrepreneurship: practice and principles. New York: HarperCollins Publishers.

Drew, S. (2006). Building technology foresight: using scenarios to embrace innovation. European Journal of innovation management, 9(3), 241-257.

Dobni, C. B. (2008). Measuring innovation culture in organizations. European Journal of Innovation Management, 11(4), 539-559.

Doolin, B., \& Troshani, I. (2007). Innovation diffusion: a stakeholder and social network view. European Journal of Innovation Management, 10(2), 176-200.

Duin, P., \& Ortt, J. (2008). The evolution of innovation management towards contextual innovation. European Journal of Innovation, 11(4), 522-538.

Evans, K., Fang, E., \& Palmatier, R. (2008). Influence of customer participation on creating and sharing of new product value. Journal of Academy of Marketing Science, 36(3), 322-336.

Ferro, A., Rohrich, S., Quadros, R., \& Gavira, M. (2007). Gestão da inovação tecnológica: uma análise da aplicação do funil de inovação em uma organização de bens de consumo. Revista de Administração Mackenzie, 8(1).

Fries, L. (2010). Tinkenring with market actors. In: Araujo, L.; Finch, J.; Kjellberg, H. (ed.). Reconnecting marketing to markets. Oxford: Oxford University Press. 138-157.

Revista de Administração e Inovação, São Paulo, v. 10, n.2, p.238-261, abr./jun. 2013. 
Garcez, M., \& Wright, J. (2010). Estudo de modelos de previsão tecnológica aplicados à substituição de embalagens de refrigerantes para o mercado brasileiro. Revista de Administração da Universidade de São Paulo, 45(3).

Granovetter, M. (1985). Economic Action and Social Structure: The Problem of Embeddedness. American Journal of Sociology, 91(3) 481-510.

Golfetto, F., \& Rinallo, D. (2006). Representing markets: the shaping of fashion trends by French and Italian fabric companies. Industrial Marketing Management, 35(7), 856-869.

Gonçalves Filho, C., Gonçalves, C. A., \& Pardini, D. J. (2008). O impacto da inovação e da gestão do conhecimento de marketing no desempenho de novos produtos no mercado. Revista de Administração e Inovação, 5(2), 5-24.

Gotze, E., Prange, C., \& Uhrovska, I. (2009). Childrens's impact on innovation decision making. European Journal of Marketing, 43(1/2), 264-295.

Guimarães, V., \& Monaco, S. (2007). Creativity and innovation in the context of team-work: an evaluation in the self-management cells and quality control circles of Ambev S.C. Branch. Brazilian Administration Review, 4(1), 21-34.

Hagberg, J., \& Kjellberg, H. (2010). Who performs marketing? Dimensions of agential variation in market pratice. Industrial Marketing Management, 39(6), 1028-1037.

Håkansson, H., \& Waluszewski, A. (2007). Knowledge and innovation in business and industry: The importance of using others. London: Routledge.

Hyssalo S., \& Stewart, J. (2008). Intermediaries, users and social learning in thechnological innovation. International Journal of Innovation Management, 12(3), 295-325.

Hoholm, T., \& Araujo, L. (2011). Studying innovation process in real-time: the promises and challenges of etnography. Industrial Marketing Management, 40(6), 933-939.

Hooley, G., Perin, M., \& Sampaio, C. (2007). Impacto dos recursos da empresa na performance de inovação. Revista de Administração de Empresas, 47(4), 46-58.

Jong, J., \& Hartog, D. (2007). How leaders influence employees' innovative behaviour. European Journal of Innovation Management, 10 (1), 41-64.

Joshi, A. (2008). Salesperson influence on product development: insights from a study of small manufacturing organizations. Journal of Marketing, 72(2), 94-107.

Kalling, T. (2007). The lure of simplicity: learning perspective on innovations. European Journal of Innovation Management, 10(1), 65-89.

Kalwani, M., Min, S., \& Robinson, W. (2006). Market pioneer and early follower survival risks: a contingency analysis of really new versus incrementally new product-markets. Journal of Marketing, 70(1), 15-33.

Revista de Administração e Inovação, São Paulo, v. 10, n.2, p. 238-261, abr./jun. 2013. 
Kjellberg, H., \& Helgesson, C. (2006). Multiple versions of markets: multiplicity and performativity in market pratice. Industrial Marketing Management, 35(7), 839-855.

Kjellberg, H., \& Helgesson, C. (2007a). On the nature of markets and their practices. Marketing Theory, 7(2), 137-162.

Kjellberg, H., \& Helgesson, C. (2007b). The mode of exchange and shaping of markets: distributor influence in the Swedish post-war food industry. Industrial Marketing Management, 36(7), 861-878.

Kjellberg, H. (2010). Marketing on trial: the SAS EuroBonus case. In: Araujo, L., Finch, J., \& Kjellberg, H. (ed.). Reconnecting marketing to markets. Oxford: Oxford University Press.

Klink, R. R., \& Athaide, G. A. (2010). Consumer Innovativeness and the Use of New versus Extended Brand Names for New Products. Journal of Product Innovation Management, 27(1), 23-32.

Kohli, A., \& Jaworski, B. (1990). Marketing orientation: the construct, research propositions and management implications. Journal of Marketing, 54(2), 1-18.

Laforet, S. (2008). Size, strategic, and market orientation affects on innovation. Journal of Business Research, 61(7), 753-764.

Luckas, B. A., \& Ferrell, O. C. (2000). The effect of market orientation on product innovation. Journal of The Academy of Marketing Science, 28(2), 239-247.

Lutz, A., Talke, K., Salomo, S., \& Wieringa, J. E. (2009). What about design newness? Investigating the relevance of a neglected dimension of product innovativeness. Journal of Product Innovation Management, 26(6), 601-615.

Macgregor, S. P. (2006). Supporting new product creation in the Mondragón Valley. European Journal of Innovation Management, 9(4), 418-443.

Merrilees, B., \& Miller, D. (2008). Principles of corporate rebranding. European Journal of Marketing, 42(5/6), 537-552.

Meirelles, D., \& Pinto, L. (2008). Comportamento Inovador e Desempenho das Pequenas Empresas Empreendedoras do Setor de Serviços de Alimentação. Anais do 3ES.

Miguel, L., \& Teixeira, M. (2009). Valores organizacionais e criação do conhecimento organizacional inovador. Revista de Administração Contemporânea, 13(1), 35-56.

Moretti, S. L. do A., Moysés Filho, J. E., \& Pereira, R. D. A. (2010). Inovação no desenvolvimento de produto para o mercado de sobremesas prontas: o caso da Laffriolée. Revista de Administração $e$ Inovação, 7(1), 22-40.

Neyland, D., \& Simakova, E. (2010). Trading bads and goods. In: Araujo, Luis; Finch, John; Kjellberg, Hans (Eds.). Reconnecting marketing to markets. Oxford: Oxford University Press.

Revista de Administração e Inovação, São Paulo, v. 10, n.2, p.238-261, abr./jun. 2013. 
Paladino, A. (2007). Investigating the drivers of innovation and new product success: a comparison of strategic orientations. Journal of Product Innovation Management, 24(6), 534-553.

Pereira, D., Pinto, M., \& Santos, L. (2007). Caracterização de perfis de consumidores quanto à disposição de adoção de produtos e serviços baseados em tecnologia a partir da utilização do método de grade of membership (gom). Revista Eletrônica de Administração, 13(2), 1-22.

Perez, G., \& Zwicker, R. (2010). Fatores determinantes da adoção de sistemas de informação na área de saúde: um estudo sobre o prontuário médico eletrônico. Revista de Administração Mackenzie, 11(1), 174-200.

Porto, G., Pacagnella, A., Kannebley, S., Silva, S., \& Salgado, A. (2010). Influências no desempenho inovador do setor de bens de capital: uma análise na indústria paulista. Revista Eletrônica de Administração, 16(2), 52-75.

Recwitz, A. (2002). The status of the "material" in theories of culture: from "social structure" to "artefacts". Journal for the Theory of social behaviour, 32(2), 195-207.

Sassatelli, R. (2007). Consumer culture: history, theory and politics. USA: Sage.

Sheth, J.N., Gardner, D. M., \& Garrett, D. E. (1998). Marketing Theory: Evolution and Evaluation. New York: John Wiley \& Sons.

Smith, M. et al. (2008). Factors influencing an organisation's ability to manage innovation: a strutured literature review and conceptual model. International Journal of Innovation Management, 12(4), 655676.

Silva, K., Zawislak, P., \& Carvalho, J. (2008). Estratégia de inovação: o caso da Muri Linhas de Montagem. Revista de Administração da Universidade de São Paulo, 43(2), 152-161.

Sorescu, A., \& Spanjol, J. (2008). Innovation's Effect on Firm Value and Risk: Insights from Consumer Packaged Goods. Journal of Marketing, 72(2), 114-132.

Stokes, D. (2000). Putting entrepreneurship into marketing: the process of entrepreneurship/marketing interface: a review of the evidence. Journal of Research in Marketing \& Entrepreneurship, 2(1), 1-16.

Swedberg, R. (2003). Principles of economic sociology. Princeton: Princeton University Press.

Smelser, N. J., \& Swedberg, R. (2005). Introducing Economic Sociology. The Handbook of Economic Sociology, Second Edition. Princeton.

Van de Ven, A. (1986). Central problems in the management of innovation. Management Science, 32(5), 590-607.

Wright, R. F. (2002). A review of the four prominent schools of thought. Journal of Advertising History, Special issue.

Revista de Administração e Inovação, São Paulo, v. 10, n.2, p. 238-261, abr./jun. 2013. 


\title{
MARKET PRACTICES AND INNOVATION: FORGOTTEN DIMENSIONS
}

\begin{abstract}
Developing a literature review of marketing and innovation we can see that innovation is focused only on the internal management of the organization. However, we should understand that markets are dynamic and complex and that various actors who have different beliefs and values work together through practices that shape those markets. Innovations allow destabilize structures that are balanced, generating externalities and new modes of exchange are defined, as well as new rules and regulations have to be created. These new definitions occur through practices and representations conducted by agents with expertise in marketing, which interfere in the actions of other agents. In this sense, this study aims to present how innovation has been approached by researchers to build the knowledge of the area, particularly demonstrating how dimensions present in the innovation process have been neglected. The discussion was supported by extensive review of national and international literature on innovation, by means of an analysis of 1102 articles published in journals and conference proceedings. As a result, the article presents theoretical propositions that can contribute to a more holistic view of the phenomenon, from both managerial and theoretical point of view.
\end{abstract}

Key words: Marketing management; Market practices; Innovation process.

Data do recebimento do artigo: 05/01/2012

Data do aceite de publicação: 02/06/2013

Revista de Administração e Inovação, São Paulo, v. 10, n.2, p.238-261, abr./jun. 2013. 